\title{
The Design of Geo-Spatial Metadata Architecture for Digital Boundary
}

\author{
Junxi Zhao ${ }^{\mathrm{a}}$, Jing Zhang ${ }^{\mathrm{a}}$, Yibing Cao ${ }^{\mathrm{a}}$ \\ ${ }^{\text {a }}$ Institute of Geo-Spatial Information, Information Engineering University, KeXue Street NO.62, ZhengZhou, China - \\ jxzhao@aliyun.com, 1040256713@qq.com, cao_yibing@126.com
}

KEY WORDS: Geo-spatial metadata; Field specific metadata; Digital Boundary; Architecture framework

\begin{abstract}
:
This paper analyzes the significance of geospatial metadata technology in the "“'Digital Boundary" " project. In the study of boundary data types and characteristics, the architecture framework of geospatial metadata is designed. It lays foundation for establishing detailed content of all kinds of metadata as the next step.
\end{abstract}

\section{INTRODUCTION}

China's land borders a total length of over $22,000 \mathrm{~km}$, with 14 countries bordering, therefore the border situation is very complex. Border job is a long-term and important task in China's diplomatic work, which directly affects China's sovereignty $\backslash$ territorial integrity, national unity and the border region's economic development, social stability and people's living ${ }^{[1-2]}$. Since the mid-1990s, Geographic information technology was use for the land border operations in China. It is based on the boundary geospatial databases, and take delimitation, demarcation, joint inspection and other systems as the core, which realized China's land border integrated management of massive data. However, this systems only play a role in some specific work, because of lacking unified technical standard and design, it is difficult to share data resources between different systems, so it can't meet the needs of the boundary information management and application neither.

The aim of "Digital Boundary" project construction is to realize rapid access and integrated management of the various boundary information, to implement the boundary information integration and information sharing between different departments, to achieve the comprehensive analysis and auxiliary decision-making for the boundary information application, to provide data resources and information system security for effective application of boundary information. One of the major goals of the "Digital Boundary" project is to develop a unified technical norms and standards. The technical specifications and standards can be used to capture and integrate various boundary information resources, to design and build "Digital Boundary" database, to achieve a variety of boundary data information management and data sharing. The 'design of geographical spatial metadata system in "Digital Boundary" is the key to decide whether it ultimately achieve the goal.

Metadata is one of the six major support technologies of digital earth, become the key of digital earth construction can be successful and practical ${ }^{[3]}$. As well, Geospatial metadata technology in "Digital Boundary" is also determines whether the "Digital Boundary " project would ultimately to be success.

"Digital Boundary" metadata should belong to the field of special metadata classified, according to the application domain. At present the study of metadata in the field of "Digital
Boundary" is still rare. It is according to the characteristics of the "Digital Boundary" project construction , It can be used for "Digital Boundary" Project Implementation. It is extended based on the field of Shared metadata and metadata on the premise of meet the needs of the project ${ }^{[4]}$. Sharing metadata is a unified framework of many areas metadata. It is used for guiding all areas to develop their metadata; Fielding metadata is based on the characteristics of their field data resources, and on the basis of Sharing metadata to determine various fields the content of the metadata framework. At present, there are all kinds of metadata standard, most of them are designed for sharing metadata and fielding metadata, but in view of the "Digital Boundary", this particular project has not been corresponding domain specific metadata standards. In this paper, According to the complexity, diversity and other characteristics of the geospatial data in "digital borders" project, authors studied the geospatial metadata system framework for the border of digital. It was more easily accepted and used by the majority of users.

\section{2. "DIGITAL BOUNDARY" IN GEOSPATIAL DATA}

To design the framework of the "digital boundaries" in geospatial metadata system, you first need to study the geospatial data types and characteristics in"digital borders".

\subsection{Boundary data type}

"Digital Boundary" is a huge digital project, and the boundary information and data is very abundant. Since 60th years, through long negotiations, delimitation, demarcation, joint inspection and daily border management work, we has accumulated a large number of border management-related legal documents and maps. These include border treaties and protocols and its drawings, all relevant data segments, about have been drawn boundary and disputed boundary, markers results, photos, various agreements and joint inspection records. Currently most of our border delimitation and demarcation work has been completed, we also got a lot of information on the delimitation and demarcation of the results. In recent years, we have accumulated a large number of boundary data during the borders information digitization and the process of building applications. These existing data constitute the "digital borders" database basis ${ }^{[5]}$.

"Digital Boundary" databases include: boundary foundation geographic database, boundary landscape simulation database, 


\section{5 - 6 December 2013, Beijing, China}

boundary thematic database. The geographical spatial data, is mainly located in boundary foundation geographic database, it is includes all of the basic geographic data that we needed in "Digital Boundary" engineering construction. In addition, there are also some related to geospatial data in the boundary landscape simulation database and borders special databases ${ }^{[6-}$ 7]. The main contents of the boundaries basic geographic database is basic geographic information digital products, namely: Digital vector maps (DLG), digital raster maps (DRG), digital image map (DOM), digital elevation model (DEM), the details as followings:

\section{1 ) Digital Vector Maps}

It is the main components of the boundaries foundation geographic database, which covering all areas of the boundaries, it including roads, rivers, residential areas, transportation, contour lines and other basic geographic information.

\section{2 ) Digital Raster Maps}

It is generally used as the boundary protocol drawings $(5 \mathrm{~km}$ strip along the border range), key areas, the raster scanned maps of the dispute-prone areas and boundary markers sketch, sandbar islands sketch.

3) Digital Image Map

Resolution digital image maps for key areas, dispute-prone areas.

4) Digital Elevation Model

Covering five kilometres strip areas along the border and some key areas.

\subsection{Boundary data characteristics}

Through analysis of the data content of the basic geographic boundary, we can see geospatial data of "Digital Boundary" has the following characteristics:

1) Various of Geospatial Data

Covers: Digital vector maps (DLG), digital raster maps (DRG), digital image map (DOM), digital elevation model (DEM) and border treaty the appended drawings, protocol attached figures, boundary markers sketch, island bar sketch and so on.

2) Various Forms of Geospatial Data

"Digital Boundary" geospatial data in the form of: Digital vector maps (DLG), digital raster maps (DRG), digital image map (DOM), digital elevation model (DEM).

3) Geospatial Data are Organized by Industry Segment

Geospatial data are organized by taking the boundary segments as main line. In order to facilitate data integration management and effective use, the management of data organizations take range by partitioning, stratified by content, which partition will be mainly based on national boundaries.
4) Geospatial Data with Multiple Data Scales

"Digital Boundary" in the geospatial data involving multiple data scales [8]: "Digital Boundary" in the geospatial data involving multiple data scales [8]: 1:35,000,000 (nationwide general plan, covering areas including country, neighboringcountries), 1:400,0000 (the total figure of each industry segment, covering areas including border provinces), 1:250,000 (special area in Figure), 1:50,000 (coverage area including $5 \mathrm{~km}$ strip range along the boundary line), 1:10000 (covering built-up area of major cities in the border).

5) Great Amount of Geospatial Data

"Digital Boundary" of the great amount of data, only the 1:50000 land borders vector map are about 1,300 pieces.

\section{GEOSPATIAL METADATA SYSTEM FRAMEWORK}

\subsection{Principles of system framework design}

Based on the content and features of geospatial data in "digital borders", the principles of design digital geospatial metadata boundary system framework as follow:

1 ) System framework must be agree on the whole geospatial data system framework in "digital borders".

2 ) Geospatial metadata organization can be organized by boundary paragraph, the same as geospatial data organized in "digital borders".

3 ) Geospatial metadata system can be divided into different application layers, based on the purpose of the use of metadata.

4 ) The description of Geospatial Metadata system framework focused on data sets ( boundary map data).

5 ) Taking the applications of "Digital Boundary" in consideration, the key point of specific content of the metadata is the selection and extension of "data quality information", "data represents information", "content information" and several other meta-data subset.

6 ) Content selection of Geospatial Metadata subset must meet the "digital border" applications, but do not be too complicated.

7 ) In developing geospatial metadata standard, we should reference and follow existing national standards and industry standards areas, and modify and expand "it according to the features of digital borders" project.

\subsection{Meta-data system framework}

According to the above design principles, the geographical spatial metadata system frame design in "Digital Boundary" is shown in Figure 1. 
The International Archives of the Photogrammetry, Remote Sensing and Spatial Information Sciences, Volume XL-4/W3, 2013 ISPRS/IGU/ICA Joint Workshop on Borderlands Modelling and Understanding for Global Sustainability 2013,

5 - 6 December 2013, Beijing, China

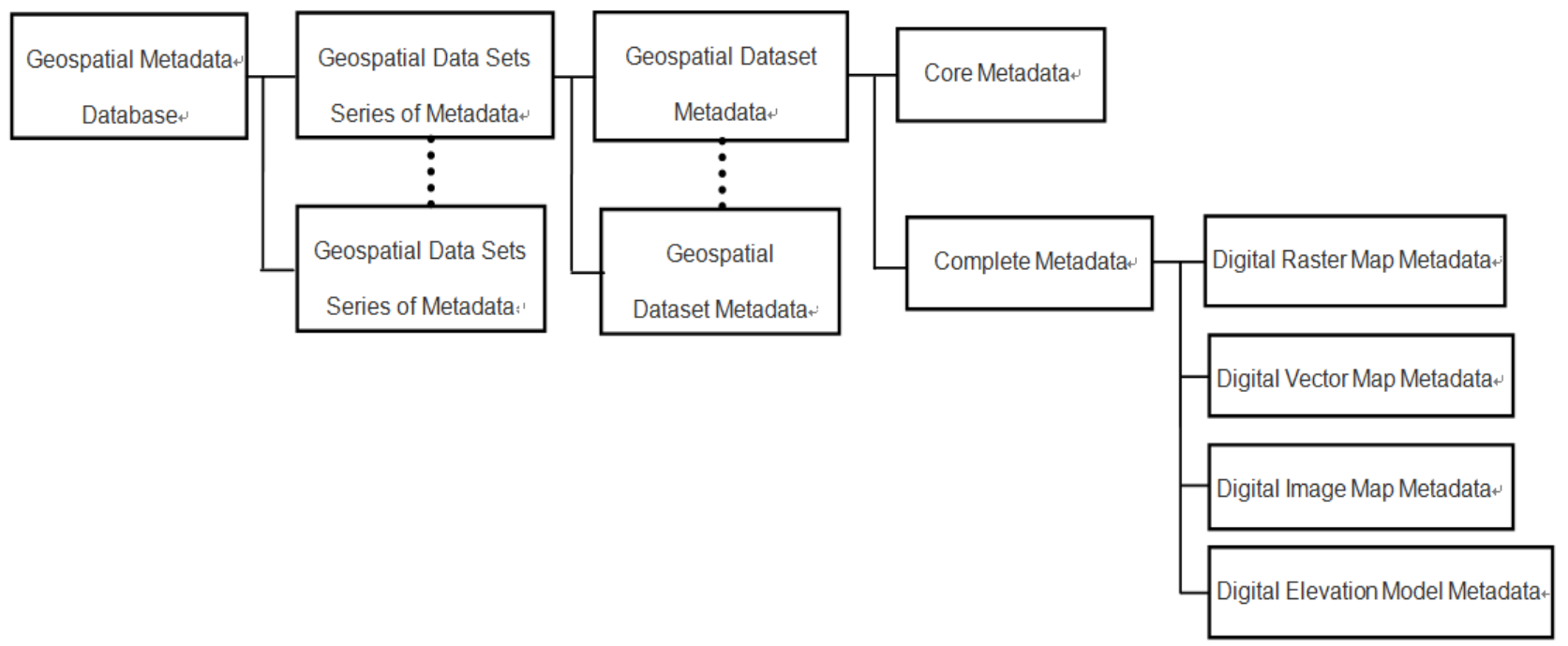

Figure 1 Geospatial Metadata system framework

System framework is a classification and hierarchical description of all geospatial metadata in "digital boundaries", various classes, all levels of metadata can be constituted an organic whole in the framework ${ }^{[9]}$. This system framework is characterized as follows:

1) According to describe differences in the object, and geospatial metadata is divided into three broad categories:

Database Metadata: also can be called "Digital Boundary" of global metadata, It is a description of the "Digital Boundary" geospatial database information, including identification of the database name, database content description, database access methods, such as the date of database update.

Dataset Series Metadata: Also can be called boundary segment metadata, is a description of industry segments, including industry segment names, logos, spatial extent, contains the number of maps.

* Dataset Metadata: Also can be called frame metadata, is a specific description information of a map sheet, including the identity, quality, content of the dataset.

2 ) Data Set of Metadata (Map Metadata) is the key to system framework and it is the key content of geospatial metadata description in "Digital Boundary".

3 ) From the application-level considerations, "Digital Boundary" Geospatial Metadata is divided into two levels:

Core Metadata: is to identify a set of data that need for the least amount of metadata entities and elements, it will no longer be subdivided into subset. It is application-oriented metadata, mainly use for data cataloguing and data discovery services.

Full Metadata: is to build a complete data collection of all the documents which needed metadata entities and elements. It is usually subdivided into several subsets. It is the metadata for the content, primarily for data identification, evaluation, extract, use and management .

4 ) Core Metadata is on the basis of fully metadata, it is generated through to the selection and tailoring of complete metadata.

5 ) Complete metadata is generated on the basis of the corresponding geospatial data products.

6 ) Corresponded to the form of "Digital Boundary" geospatial data, the form of metadata includes: digital vector map metadata, digital raster maps metadata, digital image maps metadata and digital elevation model metadata. The four kinds of data in the form of metadata content generally consistent, it include the identification information, data quality information, spatial data representation, reference to system information and issuing information . Moreover, for the same scope of space, the same scale of different data in the form of maps, the metadata standard has many same data content (such as space, space coordinate system and production units, etc.).

7 ) Digital vector map metadata, digital raster map metadata, digital image maps metadata and digital elevation model metadata are the main content form of the metadata data set.

8 ) Data set series of metadata is based on the data set of metadata. It is gotten by selection, fusion and can be further subdivided into core metadata and complete metadata.

9 ) Database Metadata is based on the metadata segment, gotten by selecting fusion obtained. It can be further subdivided into core metadata and complete metadata.

\section{CONCLUSION}

The Geospatial Metadata of the Digital Boundary" is a kind of Domain-specific metadata, which established on the basis of Shared metadata and domain metadata. In order to 
identify the content of all levels of metadata, first we should identify the metadata framework of whole digital boundary. In this paper, we analysis the data type and the characteristics of the boundary ,therefore, we design the system framework of geospatial metadata. Moreover, we take description of the metadata through classify and stratify so that lay the foundation for identifying the details of metadata of any classes.

\section{REFERENCES}

[1] Liu Hehui, Zhang Yi, Cao Yibing. Design and Key Technologies of Decision Support System for Negotiation and Delimitation of National Boundaries [J]. Geomatics \& Spatial Information Technology,2011,34(2):6 9 .

[2] Liu Hehui, Zhang Yi, Cao Yibing. Design and Application of Decision Support System for Negotiation and Delimitation of National Boundaries [J]. Geospatial Information, 2011,9(2):49 51 .

[3] Al Gore . The Digital Earth: Understanding our planet in the 21 century[R] . The OpenGIS Consortium,1998 .

[4] SDS/T 2111-2004, Basic Principles and Methods of Metadata Standardization[S] .

[5] Zhao Junxi, Zhang Yi, Song Qinghui. Design of "State Information System" Based on the PowerGIS Professional and The Design, Development , Application of GIS[M], PLA Publishing House, Beijing, 2004:149 152 .

[6] Guo Xinghua, Hua Yixin, Bai Xiaoshuang. The Design and Realization of Public Edition of China Land Boundary Information System [J]. Bulletin of Surveying and Mapping, 2008(8):56 59 .

[7] Liu Xiaolin, Hua Yixin, Zhang Xiaopeng. The Design and Realization of Boundary Information Release System [J]. Bulletin of Surveying and Mapping, 2012(2):77 78 .

[8] Guo Kui. Techniques of Geospatial Data Processing for Border Information Systems [D]. ZhengZhou : The PLA Information Engineering University Master's Degree Thesis. 2006.

[9] Zhao Junxi. Research of Geospatial Metadata for Digital Border [D]. ZhengZhou : The PLA Information Engineering University Master's Degree Thesis. 2006. 